\title{
Advances in oral health knowledge of Greek navy recruits and their socioeconomic determinants
}

\author{
Athanasios I Zavras*1, Theofilos P Vrahopoulos ${ }^{2}$, Kyriakos Souliotis ${ }^{3}$, \\ Spyros Silvestros ${ }^{2}$ and Ioannis Vrotsos ${ }^{2}$
}

Address: ${ }^{1}$ Dept. of Health Policy \& Epidemiology, Harvard School of Dental Medicine, 188 Longwood Ave., Boston, Ma. 02115, USA, ${ }^{2}$ Dept. of Periodontology, University of Athens School of Dentistry, 2 Thivon Street, Goudi, Athens, Greece and ${ }^{3}$ Dept. of Health Economics, National School of Public Health (NSPH), 196 Alexandras Ave., Athens, Greece

Email: Athanasios I Zavras* - zavras@hms.harvard.edu; Theofilos P Vrahopoulos - vrahopoulos@dent.uoa.gr; Kyriakos Souliotis - soulioti@hol.gr; Spyros Silvestros - ssylvestros@dent.uoa.gr; Ioannis Vrotsos - vrotsos@ dent.uoa.gr

* Corresponding author

Published: 19 December 2002

BMC Oral Health 2002, 2:4
Received: 27 August 2002

Accepted: 19 December 2002

This article is available from: http://www.biomedcentral.com/l472-683I/2/4

(C) 2002 Zavras et al; licensee BioMed Central Ltd. This is an Open Access article: verbatim copying and redistribution of this article are permitted in all media for any purpose, provided this notice is preserved along with the article's original URL.

\begin{abstract}
Background: The aim of the present study was to evaluate temporal changes on oral health knowledge and attitudes among two populations of young Greek males (age 19-29 years) undergoing their military training. A secondary aim was to identify socioeconomic trends that may have influenced the above.

Methods: A total of 2,764 healthy subjects (15II in 1985, and 1253 in 1996) answered a standardized questionnaire of oral health issues. Respondents were stratified in 3 groups, according to their educational status (ES); their responses were compared using the chi-square statistic. The evaluation of socioeconomic indicators utilized longitudinal data from the national Household Expenditures Survey.

Results: An overall improvement was noted in the majority of "Knowledge" and "Attitudes" variables in 1996, as compared to 1985. Education seems to play an important role in both knowledge and attitudes. These changes may also be partially attributed to a shift in consuming standards from the coverage of main needs to income disposal directed towards the achievement of better quality of life.
\end{abstract}

\section{Background}

Although there is overwhelming evidence that periodontal disease and dental caries affect the majority of the population, their prevalence and severity varying according to age, sex, race, geographic areas, socioeconomic factors, local oral as well as systemic factors and methods of oral cleaning [1], there are no reliable national statistics in Greece. The available prevalence and incidence rates for both conditions depend on limited local evidence, as explained later. Lack of disease registries, lack of a functioning national primary care system, and absence of dedicated full-time research staff at major academic institutions are some of the reasons for such luck of nationwide information. The incomplete data that currently exists as a result of scarce surveys of oral health among children [2,3], as well as adults [4-8] suggest that both, periodontal disease and dental caries exhibit an overall high prevalence. It is also evident from these studies that the level of knowledge and the overall attitude towards various oral health issues were rated as low. 
Table I: Oral health knowledge: Data and crude statistical comparisons $\left(X^{2}\right)$ between 1985 \& 1996

\begin{tabular}{|c|c|c|c|c|}
\hline \multirow[t]{2}{*}{ Year } & \multicolumn{3}{|c|}{ Educational Status } & \multirow[t]{2}{*}{ Statistic } \\
\hline & High & Medium & Low & \\
\hline QI. & \multicolumn{4}{|c|}{ Gingival bleeding considered normal (positive answer) } \\
\hline 1985 & $187(59.2 \%)$ & $397(63.7 \%)$ & $4 \mid 2(72.0 \%)$ & $X^{2}=10.8$ \\
\hline 1996 & $150(47.9 \%)$ & $289(54.9 \%)$ & $311(75.1 \%)$ & $P$-value $=0.001$ \\
\hline Q2. & \multicolumn{4}{|c|}{ Gingivitis is the cause of loosing teeth (positive answer) } \\
\hline 1985 & $102(32.3 \%)$ & $83(13.3 \%)$ & $69(12.1 \%)$ & $x^{2}=49.6$ \\
\hline 1996 & $119(38.0 \%)$ & $84(16.0 \%)$ & $147(35.5 \%)$ & $P$-value $<0.001$ \\
\hline Q3. & \multicolumn{4}{|c|}{ Chewing hard foods cause gingival bleeding (positive answer) } \\
\hline 1985 & $122(59.2 \%)$ & $218(63.7 \%)$ & 331 (72.0\%) & $X^{2}=8.4$ \\
\hline 1996 & $94(47.9 \%)$ & $170(54.9 \%)$ & $224(75.1 \%)$ & $P$-value $=0.004$ \\
\hline Q4. & \multicolumn{4}{|c|}{ Knowing what gingivitis is (positive answer) } \\
\hline 1985 & 89 (28.2\%) & $38(6.1 \%)$ & $30(5.2 \%)$ & $X^{2}=678.9$ \\
\hline 1996 & $270(86.3 \%)$ & $273(51.9 \%)$ & $170(41.1 \%)$ & $P$-value $<0.001$ \\
\hline Q5. & \multicolumn{4}{|c|}{ Toothpaste (by itself) treat gingivitis (positive answer) } \\
\hline 1985 & $108(34.2 \%)$ & $293(47.0 \%)$ & $378(66.1 \%)$ & $X^{2}=18.2$ \\
\hline 1996 & $81(25.9 \%)$ & $215(40.9 \%)$ & $248(59.9 \%)$ & $P$-value $<0.001$ \\
\hline Q6. & \multicolumn{4}{|c|}{ Toothpicks alone are sufficient to clean teeth (positive answer) } \\
\hline 1985 & $82(75.9 \%)$ & $183(29.4 \%)$ & $358(62.6 \%)$ & $X^{2}=343.1$ \\
\hline 1996 & $19(6.1 \%)$ & $42(19.5 \%)$ & $62(25.0 \%)$ & $P$-value $<0.001$ \\
\hline Q7. & \multicolumn{4}{|c|}{ Selecting the best combination of devices to clean teeth } \\
\hline 1985 & $18(5.7 \%)$ & $24(3.9 \%)$ & $10(1.7 \%)$ & $x^{2}=10.8$ \\
\hline 1996 & $92(29.4 \%)$ & 75 (14.3\%) & $32(7.7 \%)$ & $P$-value $=0.001$ \\
\hline
\end{tabular}

Table 2: Attitudes towards oral health issues and the dentist: Data and crude statistical comparisons ( $\left.X^{2}\right)$ between 1985 \& 1996

\begin{tabular}{|c|c|c|c|c|}
\hline \multirow[t]{2}{*}{ Year } & \multicolumn{3}{|c|}{ Educational Status } & \multirow[t]{2}{*}{ Statistic } \\
\hline & High & Medium & Low & \\
\hline Q9. & \multicolumn{4}{|c|}{ Not happy with present condition of teeth } \\
\hline 1985 & $142(44.9 \%)$ & 158 (25.4\%) & $386(67.5 \%)$ & $X^{2}=6.8$ \\
\hline 1996 & $100(31.9 \%)$ & $180(34.2 \%)$ & $227(54.8 \%)$ & $\mathrm{P}$-value $=0.009$ \\
\hline Q 10. & \multicolumn{4}{|c|}{ Express willingness to restore/fix dental problems } \\
\hline 1985 & $142(44.9 \%)$ & $136(21.8 \%)$ & $312(54.5 \%)$ & $X^{2}=380.5$ \\
\hline 1996 & $244(78.0 \%)$ & $378(71.9 \%)$ & $331(80.0 \%)$ & $\mathrm{P}$-value $<0.001$ \\
\hline Q. 11 & \multicolumn{4}{|c|}{ Having visited the dentist at least once } \\
\hline 1985 & 121 (38.3\%) & $173(27.8 \%)$ & $325(56.8 \%)$ & $X^{2}=901.9$ \\
\hline 1996 & $304(97.1 \%)$ & $501(95.2 \%)$ & $391(94.4 \%)$ & $P$-value $<0.001$ \\
\hline Q. 12. & \multicolumn{4}{|c|}{ Reported frequency of dental visits once or twice per year } \\
\hline 1985 & $104(32.9 \%)$ & $107(17.2 \%)$ & $59(10.3 \%)$ & $X^{2}=61.2$ \\
\hline 1996 & $166(53 \%)$ & $113(21.5 \%)$ & $81(19.6 \%)$ & $P$-value $<0.001$ \\
\hline QI3. & \multicolumn{4}{|c|}{ Visit the dentist only when a problem occurs } \\
\hline 1985 & $92(29.1 \%)$ & $286(45.9 \%)$ & $333(58.2 \%)$ & $X^{2}=0.37$ \\
\hline 1996 & $99(31.6 \%)$ & $242(46.0 \%)$ & $234(56.5 \%)$ & $P$-value $=0.54$ \\
\hline
\end{tabular}

The present study focused on how much young people knew about oral health in 1985 and then in 1996, what their intentions were and what may be some of the social and economic conditions that contribute towards this profile. Thus, primary aims of the study were a) to describe how knowledge and attitudes about oral health have changed over an eleven-year interval among young
Greek military recruits, aged 20-29; and b) to evaluate the possible impact of several social and economic trends and their role as indicators of the observed increased awareness of military recruits on issues of oral health in Greece. 


\section{Methods}

Data was collected initially in 1985; the exact same methodology was applied 11 years later, in a different group of military recruits. A total of 1511 Greek subjects aged 2029 years participated in the first survey (1985), and 1253 participated in the second survey (1996). All were healthy male recruits at the Hellenic Naval Force, undergoing their basic military training at the "Palaskas" Training Center in Elefsis, Greece. All recruits were asked to participate and were given a standardized questionnaire, administered by a team of 3 dentists - investigators; the questionnaire consisted of 13 different binary questions. Table 1 and Table 2 provide details about the questions. For example, the first question listed in Table 1 would be: "Is gingival bleeding normal ?" and the possible answers would be a) Yes, b) No and c) Do not Know. Most of the asked questions required such a response, except for those items listed on Table 2 that required the subject to answer on the frequency of visit, in which case the answer was open to any given number (continuous variable).

The estimated time to completion was 5-7 minutes. Questions were categorized in two distinct groups: the first group assessed the level of knowledge of various oral health issues and the second group assessed attitudes towards oral health as well as utilization of services statistics. All questions were simple. Although no formal validation was performed in this instrument, recruits seemed to comprehend the questions and addressed them appropriately.

Before participation, no subject had prior knowledge of the contents of the questionnaire. During the completion of the questionnaire, the researchers provided the recruits with the appropriate guidance and assistance whenever necessary, but in no way interfered and/or influenced their answers. All participants returned the answered questionnaires anonymously. To protect anonymity further, no personal identifiers were used and the answers could not be linked to their source.

In order to evaluate the potential impact of the educational status on both knowledge and attitudes of the recruits, the data collected in 1985 and again in 1996 were each stratified in 3 groups. Group A included subjects with a high educational status (HES), composed of those who hold College and University degrees, plus those with postgraduate degrees. The total number of HES trainees was 629 (316 in 1985 and 313 in 1996). Group B included subjects with a medium ES (MES), composed of High School and Lykeion graduates as well as those who graduated from Middle Technical Schools, totaling 1149 trainees (623 in 1985 and 526 in 1996). Finally, Group C was composed of 986 recruits with a low ES (LES) and includ- ed those with 6 or less years of schooling (572 in 1985 and 414 in 1996).

Data were computed and analyzed using the statistical program Stata 6.0 for Windows (Stata Corp.). Pearson's Chi-square $\left(\mathrm{x}^{2}\right)$ was utilized to test if the observed differences in oral health knowledge and attitudes between the recruit cohorts of 1985 and 1996, were statistically significant. For descriptive purposes, data are presented according to respondents' educational level; however the reported statistical comparisons relate to the crude 1985 vs. 1996 comparison. The alpha level was set to $5 \%$, and thus, $\mathrm{p}<0.05$ denotes statistical significance.

In addition to the questionnaire data, we performed an ecologic evaluation of several social and economic conditions that may impact oral health. Our analyses were based on data derived from the Household Expenditures Survey (HExS). The HExS is a nationwide survey, being conducted by The Statistical Agency of the Hellenic Republic every 5 years [9-12].

All households are eligible to participate, regardless of size, income or any other social characteristic. A multistage stratified sampling is applied, depending on the urbanization degree of the municipalities and their communes. The unified general sampling fraction (for the whole year) is $2 / 1.000$.

The Surveys record data on income of household's members, demographics, expenditures for goods and services, quantities for each reported good or service, and distribution of such items to household members. Mean expenditures per household are calculated for several groups of goods or services. The household surveys we chose to analyze were those being conducted during the years 1982 [9], 1987 [10] and 1993 [11], on a sample of 6.000 and 6.756 households, respectively.

\section{Results}

Table 1 summarizes our findings on knowledge of oral health issues; Table 2 summarizes the findings on attitudes about oral health. As mentioned earlier, tables present the data stratified according to educational status; they also provide the crude statistical comparison (chisquare, p-value) between 1985 and 1996.

Regarding knowledge about gingival bleeding (subjects were asked if they regarded gingival bleeding as normal) we notice that as the educational status decreased, the responses that gingival bleeding is normal tended to increase. In 1996 there were far fewer subjects reporting that bleeding of gums is normal than in 1985 , and this difference is statistically significant. However, the reverse trend applies to the LES subjects, although the difference be- 
Table 3: Population, Gross Domestic Product (GDP) and GDP per capita in Greece, 1985-1996

\begin{tabular}{|c|c|c|c|c|c|c|}
\hline Year & Population & $\begin{array}{c}\text { GDP } \\
\text { (in Euro) }\end{array}$ & $\begin{array}{l}\text { GDP Euro } \\
\text { per Capita }\end{array}$ & $\begin{array}{c}\text { GDP* } \\
\text { (in Euro) }\end{array}$ & $\begin{array}{l}\text { GDP* Euro } \\
\text { per Capita }\end{array}$ & $\begin{array}{c}1985-96 \\
\text { variation: } 19 \%\end{array}$ \\
\hline 1985 & 9.934 .294 & 16.409.549.523 & 1.652 & 65.014 .063 .096 & 6.544 & \\
\hline 1988 & 10.037 .037 & 26.908.346.295 & 2.681 & 69.244.328.687 & 6.899 & 5,8 \\
\hline 1991 & $|0.247 .34|$ & 47.631 .724 .138 & 4.648 & 76.652 .275 .862 & 7.480 & 8,6 \\
\hline 1994 & 10.426 .289 & 70.384 .845 .194 & 6.751 & $76.7 \mid 3.728 .540$ & 7.358 & $-2,1$ \\
\hline 1996 & 10.475 .878 & 87.850 .564 .930 & 8.386 & 81.509 .153 .338 & 7.781 & 5,9 \\
\hline
\end{tabular}

*: prices standardized for 1995

tween 1985 and 1996 in this group is not significant (Table 1).

Regarding the question about the association between loss of teeth and "gingivitis", Table 1 shows that in 1985 there was a difference between the high (HES) and low (LES) educational status (32.3\% vs. $12.1 \%)$. This difference seems to decrease in 1996. Among all three groups, a statistically significant improvement was noted in 1996 as compared to 1985 (Table 1).

Findings relating to the relationship between gingival bleeding and chewing of hard foods follow the same pattern as the first question. Bleeding of gingiva was reported as normal in 1985. In 1996, the total percentage of individuals that considered bleeding of gums normal was significantly reduced. The greatest change was noted for individuals who belong to the high (HES) educational status (from $59.2 \%$ in 1985 to $47.9 \%$ in 1996), with those in the medium (MES) following (from $63.7 \%$ to $54.9 \%$ ). However, the finding remains very prevalent among the low (LES) educational status; $72.0 \%$ in 1985 and with $75.1 \%$ in 1996 gave a positive answer that gingival bleeding is caused by hard foods (Table 1).

A very striking difference between 1985 and 1996 is presented in regards to the nature of gingivitis. Few individuals knew what gingivitis was in 1985. However, in 1996 the numbers have increased substantially. Knowledge of gingivitis has increased from $5.2 \%$ to $41.1 \%$ in LES, from $6.1 \%$ to $51.9 \%$ in MES, and from $28.2 \%$ to $86.3 \%$ for those who belong to the self-reported HES. Again, when we compare the two different strata of education we notice a substantial two-fold difference in the percentages of individuals who know what gingivitis is $(86.3 \%$ vs. $41.1 \%$ ) (Table 1).

When subjects were asked "do you believe that toothpastes alone treat gingivitis ?" significantly less subjects reported a positive answer $(\mathrm{p}<0.01)$ in 1996. Among educational levels, there were far more subjects in the LES group giving a positive answer, than those in the HES group (Table 1).

When subjects were asked, "if only a toothpick is sufficient to clean the teeth", the majority gave a positive answer in 1985. However, this trend changed in 1996; just 6.1\% of HES subjects in 1996 down from $75.9 \%$ in 1985 considered toothpicks sufficient to clean teath, the corresponding 1996 percentages for MES and Les were 19.5\% and 25\% respectively. The observed differences between 85 and 96 are statistically significant $(\mathrm{p}<0.01)$.

When recruits were asked "if they were happy with their teeth", the majority gave a negative response in 1985. A decade later, the majority reported a positive response; this change in attitude towards their dental appearance was statistically significant $(p<0.01)$. Overall, LES subjects seemed to be less satisfied with their teeth in both 1985 and 1996, as compared with HES (Table 2).

A positive attitude towards dental treatment was expressed by the great majority of recruits in 1996. Briefly, $78 \%$ of HES, $71,9 \%$ of MES and $80 \%$ of LES would like to have their dental problems treated, as compared to the 1985 rates of $44.9 \%, 21.8 \%, 54.5 \%$ respectively (Table 2 ).

Table 2 also presents data on the utilization of dental services. When asked if they had ever visited a dentist, there were more LES subjects that had visited a dentist at least once, rather than HES in 1985. In 1996, all three educational strata scored high in having visited a dentist at least once in their lifetime. Regarding the annual frequency of the dental visits, there were a significantly greater number of individuals in 1996 who reported annual or bi-annual dental visits $(\mathrm{p}<0.01)$. Especially among HES, the number of subjects who visited their dentists at least doubled between 1985 and 1996 .

As regards the behavior of visiting a dentist only when a problematic symptom exists, the 1985 vs. 1996 comparison revealed no statistical differences $(\mathrm{p}<0.54)$. Briefly, in $1985,29.1 \%$ of HES, $45.9 \%$ of MES and $58.2 \%$ of LES 
Table 4: Monthly mean expenditures (purchases) of Greek households, 1982-1994

\begin{tabular}{|c|c|c|c|c|c|c|}
\hline \multirow{2}{*}{$\begin{array}{l}\text { Year } \\
\text { Household Expenditures }\end{array}$} & \multicolumn{2}{|c|}{1982} & \multicolumn{2}{|c|}{1988} & \multicolumn{2}{|c|}{1994} \\
\hline & Euro & $\%$ & Euro & $\%$ & Euro & $\%$ \\
\hline & Euro & $\%$ & Euro & $\%$ & Euro & $\%$ \\
\hline Foodstuffs & 47,28 & 33,9 & 121,00 & 29,9 & 237,05 & 27,8 \\
\hline Alcohol \& tobacco & 4,05 & 2,9 & 13,62 & 3,4 & 30,74 & 3,6 \\
\hline Clothing \& footwear & 18,35 & 13,1 & 58,01 & 14,3 & 90,46 & 10,6 \\
\hline $\begin{array}{l}\text { Housing, Water Supply, Fuels } \\
\text { \& Lightning }\end{array}$ & 16,18 & 11,6 & 41,32 & 10,2 & 100,77 & 11,9 \\
\hline Durable domestic goods & 13,57 & 9,7 & 36,65 & 9,1 & 71,05 & 8,4 \\
\hline Education \& recreation & 8,06 & 5,8 & 27,43 & 6,8 & 61,24 & 7,2 \\
\hline $\begin{array}{l}\text { Transportation \& Communi- } \\
\text { cations }\end{array}$ & $|5,9|$ & 11,4 & 47,07 & 11,6 & 125,56 & 14,7 \\
\hline Several goods and services & 7,30 & 5,2 & 30,11 & 7,4 & 67,54 & 7,9 \\
\hline Health \& personal care & 8,94 & 6,4 & 29,48 & 7,3 & 67,07 & 7,9 \\
\hline \multicolumn{7}{|l|}{ Health (alone): } \\
\hline Hospitals & 1,16 & 17,16 & 3,12 & 15,00 & 7,79 & 16,16 \\
\hline Doctors & 2,13 & 31,58 & 7,03 & 33,84 & 13,46 & 27,92 \\
\hline Dentists & 2,15 & 31,79 & 0,01 & 34,00 & 16,55 & 34,33 \\
\hline Medications & 1,31 & 19,46 & 3,57 & 17,16 & $10,4 \mid$ & 21,59 \\
\hline Total for Health & 6,76 & 100,00 & 20,78 & 100,00 & 48,26 & 100,00 \\
\hline GRAND TOTAL & 139,63 & 100 & 404,68 & 100 & 851,49 & 100 \\
\hline
\end{tabular}

Sources : a) National Statistical Service of Greece. Household expenditure survey, 198|-82. Athens, Republic of Greece, 1983, b) National Statistical Service of Greece. Household expenditure survey, 1987-88. Athens, Republic of Greece, 1989, c) National Statistical Service of Greece. Household expenditure survey, 1993-94. Athens, Republic of Greece, 1995.

answered that they visit the office only when a dental problem occurs. In 1996, the respective results were $31.6 \%, 46 \%$ and $56.5 \%$. Among educational strata, there seemed to be a linear trend; visiting the dentist when there is a symptom was more prevalent as the educational class decreased.

To answer the question if education plays a role in knowledge or attitudes towards dental care, the results were analyzed by educational level. The stratified analysis revealed statistically significant differences $(p<0.05)$ between the three educational strata in all questions, but one. The only question in which education seemed not to play a role was the "willingness to restore teeth and fix dental problems" $(p=0.12)$. In $1985,44.9 \%$ of HES subjects and $54.5 \%$ of LES expressed the willingness to restore teeth; in 1996, $78 \%$ of HES subjects and $80 \%$ of LES expressed the willingness to restore teeth.

Table 3 presents population size, Gross Domestic Product (GDP) and GDP per capita statistics for five distinct time points from 1985 to 1996 [13].

Regarding the consuming priorities of Greek households (Table 4), the Household Expenditure Survey of 1981-82 showed that foodstuffs held the first place of the monthly mean household purchases $(33,9 \%)$, while the group of clothing and footwear followed, with a much lower percentage $(13,1 \%)$. Housing expenditures, water supply, fuels and lighting consumed $11,6 \%$ of the monthly mean household income, followed by the expenditures on transportation and communication $(11,4 \%)$. Health and personal care expenditures held the sixth place $(6,4 \%)$.

The Survey of 1987-88 showed a change in high household expenditures; the difference between the purchase for foodstuff and the one for clothing and footwear reduced. The two purchase categories followed opposite processes; the foodstuff purchases reduced while the clothing and footwear purchases increased. Health remained at the $6^{\text {th }}$ place, increasing just one percentage unit $(7,3 \%)$.

The last research on Household Expenditures, the Survey of 1993-94, presented significant changes. The clothing and footwear goods dropped to the fourth place (a reduction of more than three percentage units to $10,6 \%$ ); purchases for transportation and communication climbed in the second place, consuming $14,7 \%$ of the household income. Purchases on foodstuffs remained on the first place, although they were reduced to $27,8 \%, 6$ percentage units lower than in 1981-82. Health care purchases remained on the $6^{\text {th }}$ place; they consumed $7,9 \%$ of the household income (in comparison with $6.4 \%$ eleven years earlier). 
Table 5: Income level and expenditures for dental care in Greece, 1994

\begin{tabular}{lcccc}
\hline Strata of income levels & Mean (in Euro) & $\begin{array}{c}\text { Expenditures by income } \\
\text { level (in Euro) }\end{array}$ & Exp. - Mean (in Euro) & Exp - Mean (\%) \\
\hline I (lowest) & 16,55 & 0,51 & $-16,04$ & $-96,89$ \\
2 & 16,55 & 1,68 & $-14,87$ & $-89,82$ \\
3 & 16,55 & 1,83 & $-14,73$ & $-88,97$ \\
4 & 16,55 & 2,64 & $-13,91$ & $-84,04$ \\
5 & 16,55 & 5,48 & $-11,08$ & $-66,92$ \\
6 & 16,55 & 12,13 & $-4,42$ & $-26,71$ \\
7 & 16,55 & 12,72 & $-3,83$ & $-23,15$ \\
8 (highest) & 16,55 & 42,50 & 25,95 & 156,74 \\
\hline
\end{tabular}

Table 6: Dental manpower statistics, 1995

\begin{tabular}{lcc}
\hline \multicolumn{1}{c}{ REGION } & Dentists per 10.000 Inhabitants & Ratio of dentist to population \\
\hline Greater Athens & 16,13 & $1: 620$ \\
Rest of Central Greece \& Evia & 6,11 & $1: 1637$ \\
Peloponisos & 6,96 & $1: 1437$ \\
lonian Islands & 5,47 & $1: 1828$ \\
Eipiros & 8,07 & $1: 1239$ \\
Thesalia & 7,84 & $1: 1276$ \\
Macedonia & 10,03 & $1: 997$ \\
Thrace & 6,12 & $1: 1634$ \\
Aegean Islands & 6,35 & $1: 1575$ \\
Crete & 7,81 & $1: 1280$ \\
Greece & 10,39 & $1: 962$ \\
\hline
\end{tabular}

Source : Souliotis K. Financing healthcare services in Greece. Monography, Panteion University. Athens, 2000a

As the income increase allowed for easier coverage of the households' main needs (nutrition, clothing, housing), consuming standards changed and income disposal was propelled toward the achievement of a better life quality, which can be obtained through education (from 5.8\% rose to $7.2 \%$ ), culture, diversion and aspiration for a better health level.

At the same time, the supply of health care services grew considerably and private life and health care insurance plans appeared. However, insurance plans did not provide dental benefits. Dental care was (and still is) mainly financed through "out-of-pocket" expenses, since only $1.23 \%$ of public health care expenditures are spent on dentistry [14].

A detailed analysis of the household expenditures for health care services revealed that purchases on dental services comprised $24 \%$ of total health care purchases.

Dental expenditures assume an increasing proportion of health care, which may be due to the increase of the number of dentists, $88 \%$ of whom practice solo private dentistry, the increase of expensive prosthetic operations and the significant decline of the public role in the provision of dental coverage. In a national level in 1998, dentistry accounted for the majority of all private health care expenditures (34\%); hospital care accounted for $12,4 \%$, medical care accounted for 31,4\%, pharmaceutical care accounted for $15,4 \%$ and the rest services accounted for $6.9 \%[12]$.

The mode of healthcare utilization in Greece is strongly depended on place of residence. Table 5 presents the regional flow of patients from less-developed prefectures to well-developed regions, such as to Athens (the capital of Greece), Macedonia (especially to the city of Thessaloniki) and to Ipiros. Personal and regional income inequalities seem to influence both the demand and the supply of dental services. Table 6 presents the regional distribution of dentists and physicians. Finally, Table 7 presents statistics about the association between income level and consumption of dental care, as a confirmation to the issues raised previously. 
Table 7: Income level and expenditures for dental care in Greece, 1994

\begin{tabular}{lcccc}
\hline Strata of income levels & Mean (in Euro) & $\begin{array}{c}\text { Expenditures by income } \\
\text { level (in Euro) }\end{array}$ & Exp. - Mean (in Euro) & Exp - Mean (\%) \\
\hline I (lowest) & 16,55 & 0,51 & $-16,04$ & $-96,89$ \\
2 & 16,55 & 1,68 & $-14,87$ & $-89,82$ \\
3 & 16,55 & 1,83 & $-14,73$ & $-88,97$ \\
4 & 16,55 & 2,64 & $-13,91$ & $-84,04$ \\
5 & 16,55 & 5,48 & $-11,08$ & $-66,92$ \\
6 & 16,55 & 12,13 & $-4,42$ & $-26,71$ \\
7 & 16,55 & 12,72 & $-3,83$ & $-23,15$ \\
8 (highest) & 16,55 & 42,50 & 25,95 & 156,74 \\
\hline
\end{tabular}

Source : Souliotis K., The role of the private sector in the Greek Health Care System. 2000b, Papazisis. Athens, Greece

\section{Discussion}

The findings presented in this paper are believed to be the first time-series report that documents oral health care knowledge and attitudes for male young Greek adults. Although no claim of representativeness can be firmly made, the study samples are composed of young males from all socioeconomic strata. The comparability of the 1985 and 1996 military cohorts is possible because of the drafting method and the allocation of recruits to training camps. Military service is mandatory for all males in Greece. Navy recruits are randomly selected to serve in the Navy vs. the Military or Airforce. In addition, recruitment practices have not changed during the last 2 decades, including no major changes in applicable laws that might have influenced the socio-economic and socio-demographic compositions of the two cohorts. Indeed, the educational stratification in 1996 is comparable with the observed stratification in 1985.

Recruitment centers are limited and accumulate recruits from diverse social backgrounds. Assignment to recruitment center is a random process. In addition, all recruits were included in the subject pool and every effort was made to include them as participants. Thus, it is unlikely that selection bias have occurred.

The participants were asked to answer simple questions, the content of which remained unknown prior to its completion. Illiterate subjects were read the questions. Although it cannot be excluded, the validity of the results is unlikely to have been compromised by bias due to differential comprehension of the questions (due to socioeconomic differences within each educational stratum).

Although data collection methods based on questionnaires present certain limitations, such as over-reporting with regard to dental knowledge, oral hygiene habits and frequency of dental visits [15], a special effort was made by the researchers to select simple and precise questions in order to ensure the validity of the different variables. Results from previous studies have shown that such self-reports are quite accurate [16,17]. Furthermore, epidemiological studies performed in Sweden, covering a 20 -year period and employing questionnaire-based data, strengthen the validity of the type of methodology used in the present investigation and may safely lead to the draw of some general conclusions [18].

As expected, education seems to play an important role in both knowledge and attitudes towards oral health. The higher the educational status, the more positive the attitude about dentistry is. Similarly, the higher the educational status, the more knowledgeable the subjects are about oral cavity health and its diseases. In addition, what becomes evident from this study is the overall improvement in knowledge and attitudes, almost a decade after the initial assessment. The statistical comparison between 1985 and 1996 confirms this notion in a sometimes emphatic fashion.

Despite the obvious improvement of the level of knowledge regarding oral health issues, there is still considerable space for improvement. Our findings are in accordance with those from epidemiological studies in Greece, in the 90's, which are indicative of a decrease in the prevalence of caries and periodontal diseases and of a higher level of dental health among younger adolescents and those belonging to higher socioeconomic strata [4$6,8,19]$. Although the emerging pattern shows a declining trend in the most common oral diseases, levels are still high and underline the need for establishing and maintaining widespread public health programs that promote health and prevent disease.

Dental attendance patterns, as they emerge from the present survey, may be the most convincing evidence that the younger generations of Greeks tend to overcome factors such as fear, superstition, etc. that may exerted a pow- 
erful negative influence on the utilization of dental services in the past $[18,20]$.

The cohort of 1985 exhibits many similarities with the cohort of 1996 (age, gender, similar social origins, multiple educational stratifications, etc), but they were found to differ significantly in their oral health knowledge and attitudes. Although it would be desirable to assess several demographic and socioeconomic variables per subject, that was not feasible. This is a limitation of the present work. Thus, these important variables cannot be evaluated directly as predictors of knowledge and attitudes. To indirectly address this concern, we compiled national statistical data from the respective period regarding social, economic and behavioral/consumer characteristics and trends of the Greek population.

Several important findings emerge from these analyses that may partially explain our observations. One would assume that the observed improvements may be attributed to changes within the family, the school, the social environment, the dental profession itself, as well as the media. Our data suggest that in these 11 years, the standard of living increased, consuming standards changed, disposable income became more and the educational level improved.

The present study identified another prevalent behavior: visiting a dentist only when a symptom occurs. The temporal comparisons indicate that such behavior has remained unchanged for a large segment of the population. Potential reasons may include negligence on behalf of the subjects, absence of national dental public health programs, and, problems with access to appropriate primary care, especially for those residing in remote and isolated areas of the country.

The issue of access to care in the Greek National Health System constitutes a well-known [21] problem with various consequences. One of the consequences is the intense regional flow of patients from less-developed prefectures to well-developed regions. In addition to personal, regional income inequalities seem to influence both the demand and the supply of dental services.

The apparent association between the frequency of emergency visits and a decreased educational status possibly underlines the excessive dental needs of subjects with a reduced educational, and possibly, socioeconomic status. Our data on income and consumption of dental care suggest a relationship between socioeconomic level and oral health. Similarly, Souliotis in 2000 [14], pointed out the correlation of the expenditures for dental care with several social and economic factors (income, profession, geographic area of habitation, etc.). In particular, the lack of public dental care services combined with peoples' conviction that, even if it is provided, the quality is low, has rendered dental care as a form of "luxury", which is a grace of specific population groups.

In summary, the present results show that the levels of knowledge and attitudes about oral health among young Greeks have improved significantly during the last decade. The improvements coincide with and reflect relevant improvements in the living standards of the Greek population, including increases in income and disposable income, and the supply of healthcare services. However, since these improvements don't have elements of universality for the Greek population and for reasons of social justice, it is necessary that concerted efforts be made to implement preventive dental measures, thereby counteracting the anticipated future dental problems, especially among socially deprived groups.

\section{Authors' contributions}

AIZ was responsible for review of economic literature, data management and statistical analysis, authoring of the text, correspondence and journal submission. TPV was responsible for review of the oral health literature, authoring and revising of the text. KS was responsible for analyzing and reporting the Household Expenditure Survey data. SS participated in survey design, literature review and field data collection. He also provided expert advise on periodontal research. IV provided expert periodontal and research advise and oversighted the project.

\section{Acknowledgements}

This work was partially supported by NIDCR/NIH, through a K23 research award to Dr. Zavras

\section{References}

I. World Health Organization Epidemiology, aetiology and prevention of periodontal diseases. Geneva: Technical Report Series 1978, 62।

2. Anagnou-Vareltzides $A$, Tsami $A$ and Mitsis F Factors influencing oral hygiene and gingival health in Greek school children. Community Dent Oral Epidemiol 1 983, I I:321-324

3. Vadiakas $G$, Tsinidou $K$ and Oulis $C$ Dental health status and treatment needs of 5- and 12-year old children in Athens, Greece. Hellenic Dental Journal 1996, 6:49-53

4. Anagnou-Vareltzides A, Tsami A, Zervogianes D and Mitsis FI Oral hygiene and gingival health in Greek airforce cadet candidates. Community Dent Oral Epidemiol 1982, 10:60-65

5. Athanassouli T, Koletsi-Kounari H, Mamai-Homata $\mathrm{H}$ and Panagopoulos $\mathrm{H}$ Oral health status of an adult population in Athens, Greece. Community Dent Oral Epidemiol 1990, 1 8:82-84

6. Diamanti-Kipioti A, Papapanou PN, Moraitaki-Tsami A, Lindhe J and Mitsis $\mathrm{F}$ Comparative estimation of periodontal conditions my means of different index systems. J Clin Periodontol 1993, 20:656-661

7. Diamanti-Kipioti A, Afentoulidis N, Moraitaki-Tsami A, Lindhe J, Mitsis $\mathrm{F}$ and Papapanou PN A radiographic survey of periodontal conditions in Greece. I Clin Periodontol 1995, 22:385-390

8. Anagnou-Vareltzides A, Diamanti-Kipioti A, Afentoulidis N, Moraitaki-Tsami A, Lindhe J, Mitsis F and Papapanou PN A clinical survey of periodontal conditions in Greece. J Clin Periodontol 1996, 23:758-763 
9. National Statistical Service of Greece Household expenditure survey, 198I-82. Athens, Republic of Greece 1983,

10. National Statistical Service of Greece Household expenditure survey, 1987-88. Athens, Republic of Greece 1989,

II. National Statistical Service of Greece Household expenditure survey, 1993-94. Athens, Republic of Greece 1995,

12. National Statistical Service of Greece Statistical yearbook of Greece, 1999. Athens, Republic of Greece 2000,

13. Souliotis K Financing healthcare services in Greece. Monography, Panteion University. Athens 2000,

14. Souliotis K The role of the private sector in the Greek Health Care System. Papazisis Athens Greece 2000,

15. World Health Organization Health research methodology. Manila: WHO Regional Office for the Western Pacific 1992,

16. Hirschman RS, Leventhal H and Glynn K The development of smoking behaviour: conceptualization and supportive cross sectional survey data. J Appl Soc Psychol 1984, 1 4:184-206

17. Åstróm AN, Jackson W and Mwahgosi IEAT Knowledge, beliefs and behavior related to oral health among Tanzanian and Ugandan teacher trainees. Acta Odontol Scand 2000, 58: I I- 18

18. Hugoson A, Koch G, Bergendal T, Hallonsten A-L, Slotte C, Thorstensson $B$ and Thorstensson $\mathrm{H}$ Oral health of individuals aged 3-80 years in Jönköping, Sweden in 1973, 1983, and 1993. Swed Dent J 1995, 19:225-241

19. Åstrǿm AN and jacobsen R Stability of dental health behaviour: a 3-year prospective cohort study of 15-, 16-, and I 8-year-old Norwegian adolescents. Community Dent Oral Epidemiol 1998, 26:129-138

20. Petridou E, Athanassouli T, Panagopoulos $\mathrm{H}$ and Revinthi K Sociodemographic and dietary factors in relation to dental health among Greek adolescents. Community Dent Oral Epidemiol 1996, 24:307-3।I

21. Kyriopoulos J and Geitona $M$ Interregional flow of patients in Greece and Europe. Exadas - Health Policy Series. Athens, Greece 1995 ,

\section{Pre-publication history}

The pre-publication history for this paper can be accessed here:

http://www.biomedcentral.com/1472-6831/2/4/prepub

Publish with Bio Med Central and every scientist can read your work free of charge

"BioMed Central will be the most significant development for disseminating the results of biomedical research in our lifetime. "

Sir Paul Nurse, Cancer Research UK

Your research papers will be:

- available free of charge to the entire biomedical community

- peer reviewed and published immediately upon acceptance

- cited in PubMed and archived on PubMed Central

- yours - you keep the copyright

Submit your manuscript here:

http://www.biomedcentral.com/info/publishing_adv.asp
BioMedcentral 